\title{
Pointwise hereditary majorization and some applications
}

\author{
Ulrich Kohlenbach \\ Fachbereich Mathematik, J.W.Goethe-Universität \\ Robert-Mayer-Str. 6-10, 6000 Frankfurt am Main, FRG
}

\begin{abstract}
A pointwise version of the Howard-Bezem notion of hereditary majorization is introduced which has various advantages, and its relation to the usual notion of majorization is discussed. This pointwise majorization of primitive recursive functionals (in the sense of Gödel's $T$ as well as Kleene/Feferman's $\widehat{P R})$ is applied to systems of intuitionistic and classical arithmetic $\left(H\right.$ and $\left.H^{c}\right)$ in all finite types with full induction as well as to the corresponding systems with restricted induction $\hat{H} \uparrow$ and $\hat{H} \uparrow^{c}$.

1) $H$ and $\hat{H} \uparrow$ are closed under a generalized fan-rule. For a restricted class of formulae this also holds for $H^{c}$ and $\hat{H} \uparrow^{c}$.

2) We give a new and very perspicuous proof that for each $\Phi^{2} \in T(\widehat{P R})$ one can construct a functional $\tilde{\Phi}^{2} \in T(\widehat{P R})$ such that $\tilde{\Phi} \alpha$ is a modulus of uniform continuity for $\Phi$ on $\left\{\beta^{1} \mid \forall n(\beta n \leq \alpha n)\right\}$. Such a modulus can also be obtained by majorizing any modulus of pointwise continuity for $\Phi$.

3) The type structure $\mathcal{M}$ of all pointwise majorizable set-theoretical functionals of finite type is used to give a short proof that quantifier-free "choice" with uniqueness

$(A C !)^{1,0}$-qf. is not provable within classical arithmetic in all finite types plus comprehension (given by the schema $(C)^{\rho}: \exists y^{0 \rho} \forall x^{\rho}(y x=0 \leftrightarrow$ $A(x))$ for arbitrary $A$ ), dependent $\omega$-choice and bounded choice. Furthermore $\mathcal{M}$ separates several $\mu$-operators.
\end{abstract}

\section{Introduction}

Howard (1973) introduced the notion " $x^{*}$ maj $x$ " where $x^{*}$ and $x$ are functionals of a finite type $\rho$ and showed that each primitive recursive functional $\Phi^{\rho}$ (in the sense of Gödel's $T$ ) can be majorized by a suitable primitive recursive functional 
$\Phi^{* \rho}$. As an application he proved that no primitive recursive functional satisfies the functional interpretation of the axiom of extensionality for objects of type 2 .

A variant of this notion with the property that each majorant majorizes itself was defined in Bezem (1985) who called this notion "strong majorization". Bezem proved that the corresponding type structure $\mathcal{M}^{s}$ of all strongly majorizable set-theoretical functionals of finite type is a model of Spector's calculus $T+B R$ of bar recursive functionals, although $\mathcal{M}^{s}$ contains discontinuous functionals.

In this paper we first introduce a pointwise version maj of Bezem's strong majorization where $x^{*} \operatorname{maj}_{\rho 0} x$ is defined by $\forall n\left(x^{*} n \operatorname{maj}_{\rho} x n\right)$. The majorizable functionals are the same for both notions since, for each $x^{*}$ which majorizes $x$ in our sense, one can compute, primitive recursively in $x^{*}$, a strong majorant $\hat{x}$ for $x$ and vice versa (2.14). In particular the type structure $\mathcal{M}^{s}$ and the corresponding type structure $\mathcal{M}$ defined by pointwise majorization coincide. However the majorization relations differ for $\rho>0$ and our modification has several advantages:

1) For types $\rho \leq 2, \operatorname{maj}_{\rho}$ can be described by the natural inequality relation $\geq_{\rho}$ (2.4,2.5). This allows mathematical applications some of which are given below. More substantial ones will follow in subsequent papers.

2) The proof that $\mathcal{M}$ is a model for $T+B R$ is technically simpler than Bezem's proof for $\mathcal{M}^{s}$ (see also the proof of 3.16 below).

3) The pointwise definition is extremely convenient for the purpose of generalizing $\mathcal{M}$ to models $\mathcal{M}^{\infty, \sigma}$ of the calculi of bar recursive functionals of infinite type $T^{\infty, \sigma}+B R$; these were introduced in Friedrich (1985) in order to carry out the functional interpretation of higher classical analysis with analytical comprehension over objects of arbitrary type (via the game quantifier translation developed in Friedrich (1984)).

The models $\mathcal{M}^{\infty, \sigma}$ will be constructed in a subsequent paper where 2) and 3) will become apparent.

In this paper, we only utilize the first property of $\mathrm{maj}$ for applications.

\section{Notation}

The set $\tilde{T}$ of finite types is defined inductively:

1) $0 \in \tilde{T}$,

2) $\rho, \tau \in \tilde{T} \Rightarrow \tau(\rho) \in \tilde{T}$. 
The subset $\tilde{P} \subset \tilde{T}$ of pure types is given by

1) $0 \in \tilde{P}$

2) $\rho \in \tilde{P} \Rightarrow 0(\rho) \in \tilde{P}$

Pure types are often denoted by natural numbers: $0(n):=n+1$. Intuitively each object of type $\tau(\rho)$ is a mapping which assigns objects of type $\rho$ to objects of type $\tau$.

In the following, we omit brackets that are uniquely determined, e.g. we write $o(o o)$ instead of $o(o(o))$.

$E-H A^{\omega}$ denotes the system of extensional intuitionistic arithmetic in all finite types as defined in Troelstra (1973),1.6.12, where only equality between objects of type 0 is primitive and higher equality is inductively defined as $z={ }_{\rho \tau} u: \equiv \forall y^{\tau}\left(z y={ }_{\rho} u y\right)$.

$W E-H A^{\omega}$ is obtained from $E-H A^{\omega}$ by replacing the extensionality axiom by a quantifier-free rule of extensionality

$$
\text { ER-qf. } \frac{A_{0} \rightarrow s={ }_{\rho} t}{A_{0} \rightarrow r[s]={ }_{\tau} r[t]},
$$

where $A_{0}$ is quantifier-free.

The corresponding systems with classical logic are denoted by $E-P A^{\omega}$ and $W E-$ $P A^{\omega}$ resp. The calculus $T$ is defined to be the quantifier-free part of $W E-H A^{\omega}$ (Troelstra (1973), 1.6.13.). The theories above all contain $0^{0}$ (zero), $S^{00}$ (successor), constants $\Pi_{\rho, \tau}, \Sigma_{\delta, \rho, \tau}$ and recursor constants $R_{\rho}$ with the defining axioms $\Pi_{\rho, \tau} x^{\rho} y^{\tau}={ }_{\rho}$ $x^{\rho}, \Sigma_{\delta, \rho, \tau} x y z=_{\tau} x z(y z)$ (where $x \in \tau \rho \delta, y \in \rho \delta, z \in \delta$ ) and

$$
\left\{\begin{array}{l}
R_{\rho} 0 y z={ }_{\rho} y \\
R_{\rho}\left(S x^{0}\right) y z={ }_{\rho} z\left(R_{\rho} x y z\right) x, \text { where } y \in \rho, z \in \rho 0 \rho .
\end{array}\right.
$$

If the constants $R_{\rho}$ are replaced by elementary recursor operators $\hat{R}_{\rho}$ characterized by

$$
\left\{\begin{array}{l}
\hat{R}_{\rho} 0 y z \underline{v}=_{0} y \underline{v} \\
\hat{R}_{\rho}\left(S x^{0}\right) y z \underline{v}={ }_{0} z\left(\hat{R}_{\rho} x y z \underline{v}\right) x \underline{v},
\end{array}\right.
$$

where $y, z$ as above and $\underline{v}=v_{1}^{\rho_{1}} \ldots v_{k}^{\rho_{k}}$ such that $y \underline{v}$ is of type 0 , and if the schema of full induction is replaced by the axiom of quantifier-free induction 
(IA)-qf. $\vdash \forall f^{1}(f 0=0 \wedge \forall x(f x=0 \rightarrow f(S x)=0) \longrightarrow \forall x(f x=0))$,

then one obtains the restricted systems $E \widehat{-H} A^{\omega} \wedge, W \widehat{E-} H A^{\omega} \wedge$, with quantifierfree part $\widehat{P R}$ due to Feferman (1977) (The functionals of $\widehat{P R}$ are essentially the primitive recursive functionals in the sense of Kleene (1959)).

Each of the systems defined so far permit us to construct a term $\lambda x . t[x]$, for each term $t^{\rho}$, such that $(\lambda x . t[x])\left(t^{\prime}\right)={ }_{\rho} t\left[t^{\prime}\right] \quad$ (see Troelstra (1973), 1.6.8).

Furthermore we need the following schemata $\left(A \in \mathcal{L}\left(E-H A^{\omega}\right)\right)$

$(A C)^{\rho, \tau}: \forall x^{\rho} \exists y^{\tau} A(x, y) \longrightarrow \exists Y^{\tau \rho} \forall x^{\rho} A(x, Y x) \quad$ (choice),

$(A C)^{\rho, \tau}$-qf. $\vdash \forall x^{\rho} \exists y^{\tau} A_{0}(x, y) \longrightarrow \exists Y^{\tau \rho} \forall x^{\rho} A_{0}(x, Y x)$,

where $A_{0} \in \mathcal{L}\left(E-H A^{\omega}\right)$ is quantifier-free (quantifier-free choice),

$\left(M P^{\omega}\right): \forall \underline{x}(A(\underline{x}) \vee \neg A(\underline{x})) \wedge \neg \neg \exists \underline{x} A(\underline{x}) \rightarrow \exists \underline{x} A(\underline{x})$,

(Markov-principle)

$\left(I P_{0}^{\omega}\right): \forall \underline{x}(A(\underline{x}) \vee \neg A(\underline{x})) \wedge(\forall \underline{x} A(\underline{x}) \rightarrow \exists \underline{y} B(\underline{y})) \rightarrow \exists \underline{y}(\forall \underline{x} A(\underline{x}) \rightarrow B(\underline{y}))$

(independence-of-premiss schema). Here $\underline{x}$ denotes a finite tupel of variables.

$" \forall x \leq_{\rho} y A "$ and " $\exists x \leq_{\rho} y A "$ are used as abbreviations for " $\forall x\left(x \leq_{\rho} y \rightarrow A\right)$ " and " $\exists x\left(x \leq_{\rho} y \wedge A\right)$ " respectively. " $\forall x ; \tilde{x} \leq_{\rho} s x A$ " stands for " $\forall x \forall \tilde{x} \leq_{\rho} s x A$ ", but " $\forall x, \tilde{x} \leq_{\rho} y A$ " stands for " $\forall x \leq_{\rho} y \forall \tilde{x} \leq_{\rho} y A$ ".

\section{Basic definitions and results about maj}

The results $2.1-2.14$ can be proved within $W \widehat{E-H} H A^{\omega} \uparrow$.

\subsection{Definition (see Bezem (1985))}

The relation $x^{*} s-\operatorname{maj}_{\rho} x\left(x^{*}\right.$ strongly majorizes $\left.x\right)$ between functionals of type $\rho$ is defined inductively as follows:

$$
\left\{\begin{array}{l}
x^{*} s-m a j_{0} x: \equiv x^{*} \geq x \\
x^{*} s-m a j_{\rho \tau} x: \equiv \forall y^{*}, y\left(y^{*} s-m a j_{\tau} y \rightarrow x^{*} y^{*} s-m a j_{\rho} x^{*} y, x y\right)
\end{array}\right.
$$


(Here $\geq$ denotes the usual primitive recursively defined inequality relation for objects of type 0$)$.

Our pointwise variant of $s-m a j$ is defined as follows

\subsection{Definition}

For $x^{*}, x$ of type $\rho, x^{*} \operatorname{maj}_{\rho} x$ is given by

$$
\left\{\begin{array}{l}
x^{*} \operatorname{maj}_{0} x: \equiv x^{*} \geq x \\
x^{*} \operatorname{maj}_{\rho 0} x: \equiv \forall n^{0}\left(x^{*} n m a j_{\rho} x n\right) \\
x^{*} \operatorname{maj}_{\rho \tau} x: \equiv \forall y^{*}, y\left(y^{*} \operatorname{maj}_{\tau} y \rightarrow x^{*} y^{*} \operatorname{maj}_{\rho} x^{*} y, x y\right) \\
(\tau \neq 0)
\end{array}\right.
$$

" $x^{*} \operatorname{maj}_{\rho} x "$ is read as " $x^{*}$ pointwise strongly majorizes $x "$.

\subsection{Remark}

Howard's definition of majorization results if the clause $x^{*} y$ in 2.1 is deleted. As a consequence of this clause in the definition of $s-m a j_{\rho}$ and $m a j_{\rho}$, one has

1) $x^{*} s-\operatorname{maj}_{\rho} x \longrightarrow x^{*} s-\operatorname{maj}_{\rho} x^{*}(\operatorname{Bezem}(1985))$ and

2) $x^{*} \operatorname{maj}_{\rho} x \longrightarrow x^{*} \operatorname{maj}_{\rho} x^{*}$ (induction on the type $\rho$ ).

\subsection{Definition}

For functionals of type $\rho$ we define a natural inequality relation $\geq_{\rho}$ by

$$
\left\{\begin{array}{l}
x_{1} \geq_{0} x_{2}: \equiv x_{1} \geq x_{2}, \\
x_{1} \geq_{\rho \tau} \quad x_{2}: \equiv \forall y^{\tau}\left(x_{1} y \geq_{\rho} x_{2} y\right) ; \quad x_{1} \leq_{\rho} x_{2}: \equiv x_{2} \geq_{\rho} x_{1} .
\end{array}\right.
$$

The following lemma establishes the strong connection between $\operatorname{maj}_{\rho}$ and $\geq_{\rho}$ : 


\subsection{Lemma}

1) $\forall x^{*}, x\left(x^{*} \operatorname{maj}_{1} x \leftrightarrow x^{*} \geq_{1} x\right)$, in particular $\forall x^{1}\left(x \operatorname{maj}_{1} x\right)$;

2) $\forall x^{*}, x\left(x^{*} \operatorname{maj}_{2} x \leftrightarrow x^{*} \geq_{2} x \wedge \forall y_{1}, y_{2}\left(y_{1} \leq_{1} y_{2} \rightarrow x^{*} y_{1} \leq_{0} x^{*} y_{2}\right)\right)$;

3) $\forall x^{*}, \tilde{x}, x\left(x^{*} \operatorname{maj}_{\rho} \tilde{x} \wedge \tilde{x} \geq_{\rho} x \rightarrow x^{*} \operatorname{maj}_{\rho} x\right)$;

4) $\forall z^{*^{\rho}}, \tilde{x}^{\rho \tau}, x^{\rho \tau}\left(\forall y^{\tau}\left(z^{*} \operatorname{maj}_{\rho} \tilde{x} y \wedge \tilde{x} y \geq_{\rho} x y\right) \rightarrow \lambda y^{\tau} . z^{*} \operatorname{maj}_{\rho \tau} x\right)$

(In $3,4 \rho, \tau \in \tilde{T}$ are arbitrary).

Proof: 1) follows immediately from the definition of pointwise majorization

$2)$ " $\rightarrow$ ": Assume $x^{*} \mathrm{maj}_{2} x$. By 1) we have $\forall y^{1}\left(\mathrm{ymaj}_{1} y\right)$ and therefore $\forall y^{1}\left(x^{*} y \geq_{0}\right.$ $x y)$, i.e. $x^{*} \geq_{2} x$. Let $y_{1} \leq_{1} y_{2}$; then, again by 1), $y_{2} \operatorname{maj}_{1} y_{1}$ which implies $x^{*} y_{2} \geq x^{*} y_{1}$.

" $\leftarrow$ ": Assume $y^{*} \operatorname{maj}_{1} y$; then by 1) $y^{*} \geq_{1} y$, and therefore, by the assumption, $x^{*} y^{*} \geq_{0} x^{*} y$. Again by the assumption one has $x^{*} y \geq_{0} x y$ and hence $x^{*} y^{*} \geq_{0}$ $x^{*} y, x y$ which implies $x^{*} \operatorname{maj}_{2} x$.

3) This is proved by induction on the type.

4) Consider first the case $\tau \neq 0$. Then from $y^{*} \operatorname{maj}_{\tau} y$ it follows by 3) and 2.3.2 that

$\left(\lambda y^{\tau} \cdot z^{*}\right)\left(y^{*}\right)=z^{*} \operatorname{maj}_{\rho} z^{*}\left(=\left(\lambda y^{\tau} \cdot z^{*}\right)(y)\right), x y$,i.e. $\lambda y^{\tau} \cdot z^{*} m a j_{\rho \tau} x$.

The case $\tau=0$ is treated analogously.

\subsection{Remark}

2.5.1,2.5.2 are not valid for Bezem's or Howard's notion of majorization. 3) and 4) hold for their notions as well.

\subsection{Notation}

$x^{*} \underline{\operatorname{maj}}_{\rho} x: \equiv \begin{cases}x^{*}={ }_{0} x & \text { if } \rho=0, \\ x^{*} \operatorname{maj}_{\rho} x & \text { otherwise. }\end{cases}$

\subsection{Lemma}

Let $\rho=\tau \rho_{k} \ldots \rho_{1}$. Then 
$x^{*} \operatorname{maj}_{\rho} x \longleftrightarrow$

$\forall y_{1}^{*}, y_{1}, \ldots y_{k}^{*}, y_{k}\left(\bigwedge_{i=1}^{k} y_{i}^{*} \underline{m a j}_{\rho_{i}} y_{i} \rightarrow x^{*} y_{1}^{*} \ldots y_{k}^{*} \operatorname{maj}_{\tau} x^{*} y_{1} \ldots y_{k}, x y_{1} \ldots y_{k}\right)$.

(This holds also for $s-m a j$ if $\underline{m a j}_{\rho_{i}}$ is replaced by $s-m a j_{\rho_{i}}$ ).

Proof: By induction on k using 2.3.

\subsection{Definition}

Let $\rho=0 \rho_{k} \ldots \rho_{1}$. Then $\operatorname{Max}_{\rho}$ is defined by

$\operatorname{Max}_{\rho}\left(x^{\rho 0}\right):=\lambda n^{0}, y^{\rho_{1}}, \ldots, y^{\rho_{k}} \cdot \max \left(x 0 y_{1} \ldots y_{k}, \ldots, x n y_{1} \ldots y_{k}\right)$,

where $\max \left(u_{1}^{0}, \ldots u_{n}^{0}\right)$ is primitive recursively defined in the usual way.

\subsection{Remark}

One easily verifies that $\operatorname{Max}_{\rho}$ can be defined in $\widehat{P R}$.

\subsection{Notation}

We abbreviate $\operatorname{Max}_{\rho}\left(x^{\rho 0}\right)$ by $x^{M}$.

\subsection{Lemma}

For each $\rho \in \tilde{T}$ one has

$x^{*} \operatorname{maj}_{\rho o} x \longrightarrow \forall m^{0}, n^{0}\left(m \geq n \rightarrow x^{*^{M}} m \operatorname{maj}_{\rho} x^{M} n, x n\right)$;

in particular $\operatorname{Max}_{\rho} \operatorname{maj}_{\rho 0(\rho 0)} \operatorname{Max}_{\rho}$.

Proof: Let $\rho=0 \rho_{k} \ldots \rho_{1}, x^{*} \operatorname{maj}_{\rho 0} x$ and $y_{i}^{*} \underline{m a j}_{\rho_{i}} y_{i}(i=1, \ldots, k)$. Using only quantifier-free induction $(I A)$-qf. on $\mathrm{m}$, one proves that

$\forall m\left(\forall n \leq m\left(x^{*^{M}} m y_{1}^{*} \ldots y_{k}^{*} \geq_{0} x^{M} n y_{1} \ldots y_{k}, x n y_{1} \ldots y_{k}\right)\right)$.

By 2.8 and 2.3 this implies the lemma.

\subsection{Definition}

By $M A X$ we denote the set of all terms of $\widehat{P R}$, which are constructed from only $O^{0}, S^{00}, \Pi, \Sigma$ and $\operatorname{Max}_{\rho}$. 


\subsection{Proposition}

For each $\rho \in \tilde{T}$, there are effectively closed terms $s_{\rho}$ and $t_{\rho} \in M A X$ of type $\rho \rho$ such that

$\forall x^{*}, x\left(\left(x^{*} s-\operatorname{maj}_{\rho} x \rightarrow s_{\rho} x^{*} \operatorname{maj}_{\rho} s_{\rho} x, x\right) \wedge\left(x^{*} \operatorname{maj}_{\rho} x \rightarrow t_{\rho} x^{*} s-\operatorname{maj}_{\rho} t_{\rho} x, x\right)\right)$.

\section{Proof:}

Induction on the type $\rho: \rho=0: s_{0}:=t_{0}:=\lambda x^{0} . x$.

$\rho=\delta 0: s_{\delta 0} x^{\delta 0}:=\lambda n^{0} \cdot s_{\delta}(x n), t_{\delta 0} x^{\delta 0}:=\lambda n^{0} \cdot t_{\delta}\left(x^{M} n\right):$

(i) Assume $x^{*} s-\operatorname{maj}_{\delta 0} x$. We show that $s x^{*} \operatorname{maj}_{\delta 0} s x, x$, i.e.

(*) $\forall n\left(\left(s x^{*}\right) n m a j_{\delta}(s x) n, x n\right)$. The assumption implies

$\forall n\left(x^{*} n s-\operatorname{maj}_{\delta} x n\right)$. The induction hypothesis applied to $s_{\delta}$ yields (*).

(ii) Assume $x^{*} \operatorname{maj}_{\delta 0} x$. We have to show: $t\left(x^{*}\right) s-\operatorname{maj}_{\delta 0} t(x), x$.

By 2.12, the assumption, and 2.3.2, one has

$\forall m^{0}, n^{0}\left(m \geq n \longrightarrow x^{*^{M}} \operatorname{mmaj}_{\delta} x^{*^{M}} n, x^{M} n, x n\right)$

and hence, by the induction hypothesis applied to $t_{\delta}$

$\forall m, n\left(m \geq n \rightarrow t_{\delta}\left(x^{*^{M}} m\right) s-\operatorname{maj}_{\delta} t_{\delta}\left(x^{*^{M}} n\right), t_{\delta}\left(x^{M} n\right), x n\right)$.

$\frac{\rho=\delta \tau(\tau \neq 0)}{s_{\delta \tau} x^{\delta \tau}:=\lambda y^{\tau} \cdot s_{\delta}\left(x\left(t_{\tau} y\right)\right),}$

$t_{\delta \tau} x^{\delta \tau}:=\lambda y^{\tau} \cdot t_{\delta}\left(x\left(s_{\tau} y\right)\right)$.

(i) Assume $x^{*} s-\operatorname{maj}_{\delta \tau} x$. We show that $s x^{*} \operatorname{maj}_{\delta \tau} s x$, $x$, i.e.

$\forall y^{*}, y\left(y^{*} \operatorname{maj}_{\tau} y \longrightarrow s x^{*} y^{*} \operatorname{maj}_{\delta} s x^{*} y, s x y, x y\right)$ :

$y^{*} \operatorname{maj}_{\tau} y \stackrel{\text { ind.hypoth. }}{\longrightarrow} t_{\tau} y^{*} s-m a j_{\tau} t y, y \stackrel{\text { assumpt.2.3.1 }}{\longrightarrow}$

$x^{*}\left(t y^{*}\right) s-\operatorname{maj}_{\delta} x^{*}(t y), x(t y), x y \stackrel{\text { ind.hypoth. }}{\longrightarrow}$

$s_{\delta}\left(x^{*}\left(t y^{*}\right)\right) \operatorname{maj}_{\delta} s_{\delta}\left(x^{*}(t y)\right), s_{\delta}(x(t y)), x y$.

(ii) $x^{*} \operatorname{maj}_{\delta \tau} x \rightarrow t x^{*} s-\operatorname{maj}_{\delta \tau} t x, x$ : The proof is similar to the proof of (i) above.

\subsection{Proposition}

1) Let $t^{\tau}\left[x_{1}^{\rho_{1}}, \ldots, x_{k}^{\rho_{k}}\right] \in T$ be a term whose free variables $x_{i}$ are all of type $\rho_{i} \leq 1$. Then one can effectively compute a term $t^{*^{\tau}}\left[x_{1}, \ldots, x_{k}\right] \in T$ having at most the free variables of $t$ such that

$$
W E-H A^{\omega} \vdash \forall x^{\rho_{1}}, \ldots, x^{\rho_{k}}\left(t^{*}\left[x_{1}, \ldots, x_{k}\right] \operatorname{maj}_{\tau} t^{*}\left[x_{1}, \ldots, x_{k}\right], t\left[x_{1}, \ldots, x_{k}\right]\right) .
$$


In particular, for each closed term $t^{\tau} \in T$, there exists a corresponding closed term $t^{* \tau} \in T$ such that

$W E-H A^{\omega} \vdash t^{*} \operatorname{maj}_{\tau} t^{*}, t$.

2) For $\left.t^{\tau}\left[x_{1}, \ldots, x_{k}\right] \in \widehat{P R}(M A X), 1\right)$ holds with $t^{* \tau} \in \widehat{P R}(M A X)$ provable in $W E \widehat{-} H A^{\omega} \uparrow$.

\section{Proof:}

1) We show that for each constant $c^{\tau} \in T$ there exists a closed term $c^{* \tau} \in T$ such that $W E-H A^{\omega} \vdash c^{*} \operatorname{maj}_{\tau} c$ (Since $x_{i} \operatorname{maj}_{\rho_{i}} x_{i}$ for $\rho_{i} \leq 1$, and $t_{1}^{*} \operatorname{maj}_{\rho \tau} t_{1} \wedge t_{2}^{*} \underline{m a j}_{\tau} t_{2} \rightarrow t_{1}^{*} t_{2}^{*} \operatorname{maj}_{\rho} t_{1} t_{2}$, this implies the proposition).

It is trivial that $0^{0} \operatorname{maj}_{0} 0^{0}$ and $S \operatorname{maj}_{00} S$. By $2.8 \Pi_{\rho, \tau} \operatorname{maj}_{\rho \tau \rho} \Pi_{\rho, \tau}$ and for $\rho \neq 0 \Sigma_{\delta, \rho, \tau} \operatorname{maj} \Sigma_{\delta, \rho, \tau}$. Using 2.8 and 2.12 one shows that $\Sigma_{\delta, 0, \tau}^{*}:=\lambda x, y, z \cdot(x z)^{M}(y z) \operatorname{maj} \Sigma_{\delta, 0, \tau}$.

Ad $R_{\rho}$ : (i) $\rho \neq 0$ : By induction one easily shows that $\forall x^{0}\left(R_{\rho} x \operatorname{maj} R_{\rho} x\right)$ and therefore $R_{\rho} \operatorname{maj} R_{\rho}$.

(ii) $\rho=0: R_{0}^{*}:=\lambda x, y, z \cdot R_{0} x y\left(z^{M}\right)$. By induction and 2.12 it follows that $\forall x\left(R_{0}^{*} x \operatorname{maj} R_{0} x\right)$.

2) Define $\hat{R}_{\rho}^{*}:=\lambda x, y, z, \underline{v} \cdot \hat{R}_{\rho} x y\left(z^{M}\right) \underline{v}$.

Assume $\rho=0 \rho_{k} \ldots \rho_{1}, y^{*} \underline{m a j} y, z^{*} \operatorname{maj} z$ and $v_{i}^{*} \underline{m a j}_{\rho_{i}} v_{i}(1 \leq i \leq k)$ : By $(I A)-q f$. using 2.12 one shows

$\forall x\left(\hat{R}_{\rho}^{*} x y^{*} z^{*} \underline{v}^{*} \geq_{0} \hat{R}_{\rho}^{*} x y z \underline{v}, \hat{R}_{\rho} x y z \underline{v}\right)$. By 2.8 this yields $\hat{R}_{\rho}^{*} \operatorname{maj} \hat{R}_{\rho}$. It is clear that $\hat{R}_{\rho}^{*} \in \widehat{P R}$, since $\lambda z^{\rho 0} . z^{M} \in \widehat{P R}(2.10)$.

For $M A X$ the proposition follows from 2.12.

\subsection{Definition}

The extensional type structure $\mathcal{M}$ of all hereditarily pointwise strongly majorizable set-theoretical functionals of finite type is defined as

$$
\left\{\begin{array}{l}
M_{0}:=\omega, \quad n \operatorname{maj}_{0} m:=n \geq m \wedge n, m \in \omega \\
x^{*} \operatorname{maj}_{\rho 0} x: \equiv x^{*}, x \in M_{\rho}^{M_{0}} \wedge \forall n\left(x^{*} n \operatorname{maj}_{\rho} x n\right), \\
x^{*} \operatorname{maj}_{\rho \tau} x: \equiv x^{*}, x \in M_{\rho}^{M_{\tau}} \wedge \forall y^{*}, y \in M_{\tau}\left(y^{*} \operatorname{maj}_{\tau} y \rightarrow x^{*} y^{*} \operatorname{maj}_{\rho} x^{*} y, x y\right) \\
(\tau \neq 0) \\
M_{\rho \tau}:=\left\{x \in M_{\rho}^{M_{\tau}} \mid \exists x^{*} \in M_{\rho}^{M_{\tau}}: x^{*} \operatorname{maj}_{\rho \tau} x\right\} \quad(\rho, \tau \in \tilde{T}) .
\end{array}\right.
$$


(Here $M_{\rho}^{M_{\tau}}$ denotes the set of all total set-theoretical mappings from $M_{\tau}$ into $\left.M_{\rho}\right)$.

$\mathcal{M}:=\bigcup_{\rho \in \tilde{T}} M_{\rho}$.

\subsection{Remark}

$x^{*} \operatorname{maj}_{\rho} x \rightarrow x \in M_{\rho} \wedge x^{*} \operatorname{maj}_{\rho} x^{*} \rightarrow x^{*}, x \in M_{\rho}$ (induction on $\rho$ ).

Similar to the proof of 2.15 one shows

\subsection{Proposition}

$\mathcal{M} \models T$.

\subsection{Remark}

By the proof of 2.14 it is clear that $\mathcal{M}=\mathcal{M}^{s}$, where $\mathcal{M}^{s}$ is Bezem's type structure, which is based on $s-$ maj instead of maj (see Bezem (1985)).

\section{Applications}

Let $A(\alpha, n) \in \mathcal{L}\left(E-H A^{\omega}\right)$ be a formula whose free variables are all of type 0,1 . The usual fan-rule for systems such as $H:=E-H A^{\omega}, W E-H A^{\omega}$ in the literature (see e.g. Troelstra $(1974),(1977))$ states that

$$
(*)\left\{\begin{array}{l}
H \vdash \forall \alpha^{1} \exists n^{0} A(\alpha, n) \Rightarrow \\
H \vdash \forall \beta ; \alpha \leq_{1} \beta \exists m^{0} \forall \gamma \leq_{1} \beta\left(\bar{\gamma}\left(t_{M} \beta\right)={ }_{0} \bar{\alpha}\left(t_{M} \beta\right) \rightarrow A(\gamma, m)\right),
\end{array}\right.
$$

where $t_{M} \in T$ is a suitable term, and $\bar{\gamma} k$ denotes a primitive recursive coding for $(\gamma 0, \ldots, \gamma(k-1)) \quad$ (see Troelstra $(1973))$

Since there are only finitely many initial sequents $\bar{\alpha}\left(t_{M} \beta\right)$ a bound can be given for the number $\mathrm{m}$ (depending only on $\beta$ ) and using closure of $H$ under choice-rule one can compute such a bound from a term $t \in T$ :

$$
(* *) H \vdash \forall \beta ; \alpha \leq_{1} \beta \exists m \leq_{0} t \beta \forall \gamma \leq_{1} \beta\left(\bar{\gamma}\left(t_{M} \beta\right)={ }_{0} \bar{\alpha}\left(t_{M} \beta\right) \rightarrow A(\gamma, m)\right) .
$$


Thus in particular, omitting the continuity part of $(* *)$, one concludes

$$
H \vdash \forall \beta ; \alpha \leq_{1} \beta \exists m \leq_{0} t \beta A(\alpha, m) \text {. }
$$

This boundedness property can be generalized from type 1 to arbitrary finite types using 2.15:

\subsection{Application (Generalized "FAN"-Rule)}

1) Let $H$ be $E-H A^{\omega}, W E-H A^{\omega}$ or $W E-H A^{\omega}+\left(M P^{\omega}\right)+\left(I P_{0}^{\omega}\right)+A C$ and $A\left(x^{1}, \tilde{x}^{\rho}, y^{\tau}\right) \in \mathcal{L}(H)$ a formula with no other free variables then $x^{1}, \tilde{x}^{\rho}$ and $y^{\tau}$. Assume that $\tau \leq 2$ and that $\rho \in \tilde{T}$ is arbitrary; let $s^{\rho 1} \in T$ be a closed term. Then the following rule holds

$$
\left\{\begin{array}{l}
H \vdash \forall x^{1} ; \tilde{x} \leq_{\rho} s x \exists y^{\tau} A(x, \tilde{x}, y) \Rightarrow \\
\exists \text { a closed term } t^{\tau 1} \in T \text { such that } \\
H \vdash \forall x^{1} ; \tilde{x} \leq_{\rho} s x \exists y \leq_{\tau} t x A(x, \tilde{x}, y) .
\end{array}\right.
$$

2) Analogous for the corresponding restricted systems $\hat{H} \uparrow$ and $\widehat{P R}$ instead of $H$ and $T$.

\section{Proof:}

1) Let $H$ be $E-H A^{\omega}$ or $W E-H A^{\omega}$ and assume $H \vdash \forall x ; \tilde{x} \leq_{\rho} s x \exists y^{\tau} A(x, \tilde{x}, y)$. Using mq-realizability (see Troelstra (1973) 3.4.2-3.4.5) one finds closed terms $t_{1}, \ldots, t_{n} \in T$ such that

$$
H \vdash \forall x, \tilde{x}\left(t_{1} x \tilde{x}, \ldots, t_{n} x \tilde{x} m q\left(\tilde{x} \leq_{\rho} s x \rightarrow \exists y^{\tau} A(x, \tilde{x}, y)\right)\right)
$$

which implies

$$
H \vdash \forall x, \tilde{x}\left(\tilde{x} \leq_{\rho} s x \rightarrow t_{1} x \tilde{x}, \ldots, t_{n} x \tilde{x} m q \exists y^{\tau} A(x, \tilde{x}, y)\right)
$$

since $\tilde{x} \leq_{\rho} s x$ is purely universal. Therefore

(i) $H \vdash \forall x ; \tilde{x} \leq_{\rho} s x A\left(x, \tilde{x}, t_{1} x \tilde{x}\right)$ 
by the definition of mq-realizability. By 2.15 we can construct closed terms $t_{1}^{*}, s^{*} \in T$ such that

$H \vdash t_{1}^{*} \operatorname{maj}_{1} \wedge s^{*} \operatorname{maj} s$. Define $t:=\lambda x \cdot t_{1}^{*} x\left(s^{*} x\right)$ if $\rho>0$ and $t:=$ $\lambda x .\left(t_{1}^{*} x\right)^{M}\left(s^{*} x\right)$ otherwise. Using 2.5.1,2.5.3,2.8 and 2.12 it follows that

$$
H \vdash \forall x ; \tilde{x} \leq_{\rho} s x\left(t x \operatorname{maj}_{\tau} t_{1} x \tilde{x}\right)
$$

and therefore by 2.5 .2

$$
\text { (ii) } H \vdash \forall x ; \tilde{x} \leq_{\rho} s x\left(t x \geq_{\tau} t_{1} x \tilde{x}\right) .
$$

(i) and (ii) imply the conclusion.

Now let $H$ be $W E-H A^{\omega}+\left(M P^{\omega}\right)+\left(I P_{0}^{\omega}\right)+A C$ and assume

$$
H \vdash \forall x ; \tilde{x} \leq_{\rho} s x \exists y^{\tau} A(x, \tilde{x}, y)
$$

By $\left(I P_{0}^{\omega}\right)$ it follows that

$$
H \vdash \forall x ; \tilde{x} \exists y^{\tau}\left(\tilde{x} \leq_{\rho} s x \rightarrow A(x, \tilde{x}, y)\right) .
$$

Using functional interpretation (Gdel (1958), Luckhardt (1973), Troelstra (1973) 3.5.10) one extracts a closed term $t_{1}^{\tau \rho 1} \in T$ such that

$$
H \vdash \forall x ; \tilde{x} \leq_{\rho} s x A\left(x, \tilde{x}, t_{1} x \tilde{x}\right),
$$

since $H \vdash F \leftrightarrow F^{D}$ for every formula $F \in \mathcal{L}(H)$, where $F^{D}$ denotes the functional interpretation of $F$.

Reasoning as before one proofs that

$$
H \vdash \forall x ; \tilde{x} \leq_{\rho} s x \exists y \leq_{\tau} t x A(x, \tilde{x}, y) .
$$

2) One has only to show the soundness of mq-realizability resp. functional interpretation for the restricted systems. This is verified by an easy modification of the corresponding proofs for $H$ since relativ to $\hat{H} \uparrow$ the axiom $(I A)-$ qf. is equivalent to

$$
\forall f, x(f 0=0 \wedge \forall y<x(f y=0 \rightarrow f(S y)=0) \rightarrow f x=0)
$$

and hence to a purely universal sentence (since the bounded quantification can be expressed in a quantifier-free form in $\hat{H} \uparrow$ ). Therefore it is mq-realized and functional interpreted by itself (up to intuitionistic logical equivalence). 


\subsection{Corollary to the proof of 3.1}

1) The proof of 3.1 generalizes immediatly to the situation where one has tupels $\underline{x}: \equiv x_{1}^{\delta_{1}}, \ldots, x_{k}^{\delta_{k}}\left(\delta_{i} \leq 1\right.$ for $\left.i=1, \ldots, k\right), \underline{\tilde{x}}: \equiv \tilde{x}_{1}^{\rho_{1}}, \ldots, \tilde{x}_{m}^{\rho_{m}}, s_{1}^{\rho_{1} \delta_{k} \ldots \delta_{1}}, \ldots, s_{m}^{\rho_{m} \delta_{k} \ldots \delta_{1}}$ and

$y_{1}^{\tau_{1}}, \ldots, y_{n}^{\tau_{n}}\left(\tau_{i} \leq 2\right.$ for $\left.i=1, \ldots, n\right)$ instead of $x^{1}, \tilde{x}^{\rho}, s^{\rho 1}, y^{\tau}$. Thus

$$
\left\{\begin{array}{l}
H \vdash \forall \underline{x} ; \tilde{x}_{1} \leq s_{1} \underline{x} \ldots \forall \tilde{x}_{m} \leq s_{m} \underline{x} \exists y_{1}^{\tau_{1}}, \ldots, y_{n}^{\tau_{n}} A\left(\underline{x}, \underline{\tilde{x}}, y_{1}, \ldots, y_{n}\right) \\
\Rightarrow \exists \text { closed terms } t_{1}, \ldots, t_{n} \in T \text { such that } \\
H \vdash \forall \underline{x} ; \tilde{x}_{1} \leq s_{1} \underline{x} \ldots \forall \forall \tilde{x}_{m} \leq s_{m} \underline{x} \exists y_{1} \leq_{\tau_{1}} t_{1} \underline{x} \ldots \exists y_{n} \leq_{\tau_{n}} t_{n} \underline{x} A\left(\underline{x}, \underline{\tilde{x}}, y_{1}, \ldots, y_{n}\right) .
\end{array}\right.
$$

The following results also generalize to finite tupels. For notational simplicity we formulate them only for tupels of length 1 .

2) If $\tau \in \tilde{T}$ is arbitrary then 3.1 holds with " $\exists y\left(t x \operatorname{maj}_{\tau} y \wedge A\right)$ " instead of " $\exists y \leq_{\tau} t x A$ ". Furthermore, if one has " $\exists y^{2} ; \tilde{y}^{\tau} A$ " $(\tau \in \tilde{T}$ arbitrary $)$ instead of " $\exists y^{2} A$ " then it is still possible to compute a bound for y: $\exists y \leq_{2} t x ; \tilde{y}^{\tau} A$.

In order to get a version of 3.1, which holds for classical arithmetic, we need the following application of functional interpretation:

\subsection{Lemma}

1) Let $s^{\rho \delta} \in T$ denote a closed term and let $A_{0} \in \mathcal{L}\left(W E-H A^{\omega}\right)$ be a quantifierfree formula whose free variables are $x^{\delta}, \tilde{x}^{\rho}$ and $y^{\tau}$ where $\delta, \rho$ and $\tau$ are arbitrary types. Then the following rule holds

$$
\left\{\begin{array}{l}
W E-P A^{\omega}+A C-\text { qf. } \vdash \forall x^{\delta} ; \tilde{x} \leq_{\rho} s x \exists y^{\tau} A_{0}(x, \tilde{x}, y) \Rightarrow \\
\exists \text { a closed term } \Phi^{\tau \rho \delta} \in T \text { such that } \\
W E-H A^{\omega} \vdash \forall x^{\delta} ; \tilde{x} \leq_{\rho} s x A_{0}(x, \tilde{x}, \Phi x \tilde{x}) .
\end{array}\right.
$$

$\Phi$ can be extracted from any given proof of the assumption with the use of functional interpretation (combined with negative translation).

2) 1) holds also for $W \widehat{E-P A^{\omega}}, \widehat{P R}$ and $W \widehat{E-} H A^{\omega} \uparrow$ instead of $W E-$ $P A^{\omega}, T, W E-H A^{\omega}$. 


\section{Proof:}

1) Assume $\rho=0 \rho_{k} \ldots \rho_{1}$.

$$
\begin{gathered}
W E-P A^{\omega}+A C-\text { qf. } \vdash \forall x^{\delta} ; \tilde{x} \leq_{\rho} s x \exists y^{\tau} A_{0}(x, \tilde{x}, y) \stackrel{\leq_{\rho}-\text { def. }_{\Longrightarrow} 2.4}{\Longrightarrow} \\
W E-P A^{\omega}+A C \text {-qf. } \vdash \forall x^{\delta} ; \tilde{x}^{\rho} \exists v_{1}^{\rho_{1}}, \ldots, v_{k}^{\rho_{k}}, y\left(\tilde{x} \underline{v} \leq_{0} s x \underline{v} \rightarrow A_{0}(x, \tilde{x}, y)\right) .
\end{gathered}
$$

Using functional interpretation (combined with negative translation), one extracts a closed term $\Phi \in T$ such that

$$
W E-H A^{\omega} \vdash \forall x ; \tilde{x} \exists v_{1}, \ldots, v_{k}\left(\tilde{x} \underline{v} \leq_{0} s x \underline{v} \rightarrow A_{0}(x, \tilde{x}, \Phi x \tilde{x}) .\right.
$$

By intuitionistic logic this implies the lemma.

2) Analogous.

\subsection{Corollary}

1) Let $A_{0}(x, \tilde{x}, y) \in \mathcal{L}\left(W E-P A^{\omega}\right)$ be a quantifier-free formula with the free variables $x^{1}, \tilde{x}^{\rho}, y^{\tau}$. Assume that $\tau \leq 2$ and that $s^{\rho 1} \in T$ is a closed term. Then the following rule holds

$$
\left\{\begin{array}{l}
W E-P A^{\omega}+A C-\text { qf. } \vdash \forall x^{1} ; \tilde{x} \leq_{\rho} s x \exists y^{\tau} A_{0}(x, \tilde{x}, y) \Rightarrow \\
\exists \text { a closed term } t^{\tau 1} \in T \text { such that } \\
W E-H A^{\omega} \vdash \forall x^{1} ; \tilde{x} \leq_{\rho} s x \exists y \leq_{\tau} t x A_{0}(x, \tilde{x}, y) .
\end{array}\right.
$$

2) Analogous for $W \widehat{E-P} A^{\omega} \wedge, \widehat{P R}$ and $W \widehat{E-} H A^{\omega} \wedge$ instead of $W E-P A^{\omega}, T$ and $W E-H A^{\omega}$.

Proof: Analogous to the proof of 3.1 using 3.3 instead of mq-realizability.

As a second application of majorization (which is in fact an application of 3.4) we show how one can extract a primitive recursive modulus of uniform continuity for a closed term $t^{2} \in T(\widehat{P R})$ from extensionality proofs for $t$. We first recall a standard proof of the extensionality of $t^{2}$ from Troelstra (1973): 
Define $x_{1}^{\rho} \approx_{\rho} x_{2}^{\rho}$ by

$$
\left\{\begin{array}{l}
x_{1} \approx_{0} x_{2}: \equiv\left(x_{1}={ }_{0} x_{2}\right) \\
x_{1} \approx_{\rho \tau} x_{2}: \equiv \forall y_{1}^{\tau}, y_{2}^{\tau}\left(y_{1} \approx_{\tau} y_{2} \rightarrow x_{1} y_{1} \approx_{\rho} x_{2} y_{2}\right)
\end{array}\right.
$$

(Troelstra (1973),2.7.2).

One easily shows that for any $t^{\rho} \in T$ built up from constants, type 1 and type 0 variables

$$
W E-H A^{\omega} \vdash t \approx_{\rho} t \text { (W.A. Howard, Troelstra (1973),2.7.3)) }
$$

and

$$
W \widehat{E-} H A^{\omega} \wedge \vdash t \approx_{\rho} t \text { if } t \in \widehat{P R} .
$$

As a corollary one gets:

\subsection{Lemma}

1) (Troelstra (1973),2.7.4 (ii)): Let $t^{2} \in T$ be a term whose free variables are of type 0 and 1 . Then

$$
W E-H A^{\omega} \vdash \forall \alpha^{1}, \beta^{1}\left(\forall n\left(\alpha n={ }_{0} \beta n\right) \rightarrow t \alpha={ }_{0} t \beta\right) .
$$

2) 1) holds also for $W \widehat{E-H} H A^{\omega} \wedge$ instead of $W E-H A^{\omega}$ if $t \in \widehat{P R}$.

\subsection{Application}

Let $t \in T(\widehat{P R})$ be as in 3.5. From any given proof of the extensionality of $t$ one can extract a modulus $t_{M}^{2} \in T(\widehat{P R})$ of uniform continuity for $t$, i.e.

$$
\begin{gathered}
W E-H A^{\omega} \vdash \forall \gamma^{1} ; \alpha, \beta \leq_{1} \gamma\left(\bar{\alpha}\left(t_{M} \gamma\right)={ }_{0} \bar{\beta}\left(t_{M} \gamma\right) \rightarrow t \alpha=_{0} t \beta\right) \\
\left(W \widehat{E-} H A^{\omega} \wedge \vdash(\ldots)\right) .
\end{gathered}
$$

The free variables of $t_{M}$ are among the free variables of $t$.

Proof: Assume that

$$
W E-H A^{\omega} \vdash \forall \gamma ; \alpha, \beta \leq_{1} \gamma\left(\forall n\left(\alpha n={ }_{0} \beta n\right) \rightarrow t \alpha={ }_{0} t \beta\right) .
$$


By 3.4 and 3.2.1 one can extract a term $\Phi \in T$ (whose free variables are among the free variables of $t$ ) such that

$$
W E-H A^{\omega} \vdash \forall \gamma ; \alpha, \beta \leq_{1} \gamma\left(\forall n \leq_{0} \Phi \gamma\left(\alpha n=_{0} \beta n\right) \rightarrow t \alpha=_{0} t \beta\right) .
$$

Therefore $t_{M} \gamma:=1+\Phi \gamma$ fulfils the claim.

Analogous for $t \in \widehat{P R}$ and $W \widehat{E-} H A^{\omega} \uparrow$ instead of $W E-H A^{\omega}$.

It should be noted that the above proof of the existence of a primitive recursive modulus of uniform continuity (provable in $W E-H A^{\omega}$ or $W \widehat{E-} H A^{\omega} \wedge$ ) does not make any reference to (the formalization of) reduction sequences or to the computability of $t$.

Another way to get a modulus of uniform continuity for $t^{2}$ which gives a slightly stronger result is to majorize a modulus of pointwise continuity $\tilde{t}^{2}$ for $t$ i.e.

$$
(+) W E-H A^{\omega} \vdash \forall \alpha^{1}, \beta^{1}\left(\bar{\alpha}(\tilde{t} \alpha)={ }_{0} \bar{\beta}(\tilde{t} \alpha) \rightarrow t \alpha=_{0} t \beta\right) .
$$

The existence of such a $\tilde{t} \in T$ for $t \in T$ is proved e.g. in Troelstra (1973) or Schwichtenberg (1973). Troelstra's proof can be modified to yield the corresponding result for $t \in \widehat{P R}$ with $\tilde{t} \in \widehat{P R}$ provable in $W \widehat{E-} H A^{\omega} \wedge$ (see Kohlenbach (1990)).

\subsection{Application}

1) Let $t^{2} \in T$ be a term whose free variables are all of type 0,1 and $\tilde{t}^{2} \in T$ be a modulus of pointwise continuity for $t$ (in the sense of $(+)$ ) whose free variables are among those of $t$. Then for any $\tilde{t}^{*} \in T$ such that $W E-H A^{\omega} \vdash \tilde{t}^{*} \operatorname{maj}_{2} \tilde{t}$ the following holds

$$
W E-H A^{\omega} \vdash \forall \gamma^{1}, \alpha^{1}, \beta^{1}\left(\forall n<\tilde{t}^{*} \gamma(\alpha n=\beta n \leq \gamma n) \rightarrow t \alpha=_{0} t \beta\right) .
$$

(By 2.15 such a $\tilde{t}^{*}$ whose free variables are among those of $\tilde{t}$ can be constructed).

2) 1) holds also with $\widehat{P R}, W \widehat{E-} H A^{\omega} \wedge$ instead of $T, W E-H A^{\omega}$.

Proof: Assume that $t, \tilde{t}, \tilde{t}^{*}$ fulfil the assumptions. By 2.5.1 it follows that

$$
W E-H A^{\omega} \vdash \forall \gamma ; \alpha \leq_{1} \gamma\left(\tilde{t}^{*} \gamma \geq_{0} \tilde{t} \alpha\right)
$$


and hence by $\tilde{t}$-definition

$$
(++) W E-H A^{\omega} \vdash \forall \gamma ; \alpha \leq_{1} \gamma ; \beta^{1}\left(\bar{\alpha}\left(\tilde{t}^{*} \gamma\right)=_{0} \bar{\beta}\left(\tilde{t}^{*} \gamma\right) \rightarrow t \alpha=_{0} t \beta\right)
$$

Now we assume that $\forall n<\tilde{t}^{*} \gamma(\alpha n=\beta n \leq \gamma n)$ and define $\hat{\alpha}:=\bar{\alpha}\left(\tilde{t}^{*} \gamma\right) * \lambda k \cdot \gamma\left(k+\tilde{t}^{*} \gamma\right)$, where

$$
(\bar{\alpha} k * \delta)(n):=\left\{\begin{array}{l}
\alpha n \text { if } n<k \\
\delta(n-k) \text { otherwise }
\end{array}\right.
$$

Clearly $\hat{\alpha} \leq \gamma$ and $\overline{\hat{\alpha}}\left(\tilde{t}^{*} \gamma\right)=\bar{\alpha}\left(\tilde{t}^{*} \gamma\right)=\bar{\beta}\left(\tilde{t}^{*} \gamma\right)$. Therefore $(++)$ applied twice (to $\hat{\alpha}, \alpha$ and $\hat{\alpha}, \beta)$ yields $t \hat{\alpha}={ }_{0} t \alpha$ and $t \hat{\alpha}={ }_{0} t \beta$.

An analogous proof can be given for the case where $T$ and $W E-H A^{\omega}$ are replaced by $\widehat{P R}$ and $W \widehat{E-} H A^{\omega} \uparrow$.

As a corollary to 3.6 or 3.7 one can derive the closure of the systems $H, \hat{H} \uparrow$ under the usual fan-rule $\left(^{*}\right) .3 .7$ allows a slight strengthening of $\left(^{*}\right)$ :

\subsection{Corollary}

1) (See also Troelstra (1977), 3.6 Remarks (ii)) Let $H$ be $W E-H A^{\omega}$ or $E-H A^{\omega}$ and $A\left(\alpha^{1}, n^{0}\right) \in \mathcal{L}\left(W E-H A^{\omega}\right)$ be a formula whose free variables are all of type 0,1 . Then the following rule holds

$$
\left\{\begin{array}{l}
H \vdash \forall \alpha \exists n^{0} A(\alpha, n) \Longrightarrow \\
\exists \text { terms } t_{M}^{2}, t^{*^{2}} \in T \text { whose free var. are among those of } A_{0} \text { without } \alpha, n \text { such that } \\
H \vdash \forall \beta^{1} ; \alpha \leq_{1} \beta \exists n \leq_{0} t^{*} \beta \forall \gamma^{1}\left(\bar{\gamma}\left(t_{M} \beta\right)=_{0} \bar{\alpha}\left(t_{M} \beta\right) \rightarrow A(\gamma, n)\right) .
\end{array}\right.
$$

2) 1) holds also for $\hat{H} \uparrow$ and $\widehat{P R}$ instead of $H$ and $T$.

\section{Proof:}

1) Assume $H \vdash \forall \alpha \exists n^{0} A(\alpha, n)$. As in the proof of 3.1 one constructs a term $t \in T$ whose free variables are among those of $A$ without $\alpha, n$ such that $H \vdash \forall \alpha A(\alpha, t \alpha)$. Let $\tilde{t}^{*}$ be as in 3.7 and $t^{*} \in T$ satisfy the condition $H \vdash t^{*} \operatorname{maj} t$. Then the corollary holds with $t_{M}:=\tilde{t}^{*}$ and $t^{*}$.

2) is proved analogously.

We conclude this paper with two applications of the type structure $\mathcal{M}$ : 


\subsection{Notation}

1) Natural numbers $n$ are coded into higher types as follows:

$$
n^{0}:=n, n^{\rho \tau}:=\lambda x^{\tau} \cdot n^{\rho}
$$

2)

$$
\begin{aligned}
& \left(\overline{y^{\rho 0}, n^{0}}\right)(k):=\left\{\begin{array}{l}
y k \text { if } k<n, \\
O^{\rho} \text { otherwise }
\end{array}\right. \\
& \left(\bar{y} n * 1^{\rho 0}\right)(k):=\left\{\begin{array}{l}
y k \text { if } k<n, \\
1^{\rho} \text { otherwise }
\end{array}\right.
\end{aligned}
$$

\subsection{Definition}

By the principle of bounded choice we mean the schema

$$
(b-A C)^{\rho, \tau}: \forall Z^{\tau \rho}\left(\forall x^{\rho} \exists y \leq_{\tau} Z x A(x, y, Z) \rightarrow \exists Y \leq_{\tau \rho} Z \forall x A(x, Y x, Z)\right) .
$$

Further notations are

$$
\begin{aligned}
& (D C)^{\rho}: \forall x^{\rho} \exists y^{\rho} A(x, y) \rightarrow \forall x^{\rho} \exists z^{\rho 0}\left(z 0={ }_{\rho} x \wedge \forall z_{1}^{0} A\left(z z_{1}, z\left(z_{1}+1\right)\right)\right) \\
& \text { (dependent } \omega \text {-choice), } \\
& (C)^{\rho}: \exists y^{0 \rho} \forall x^{\rho}\left(y x={ }_{0} 0 \leftrightarrow A(x)\right) \text { (comprehension). } \\
& b-A C:=\bigcup_{\rho, \tau \in \tilde{T}}\left\{(b-A C)^{\rho, \tau}\right\}, D C:=\bigcup_{\rho \in \tilde{T}}\left\{(D C)^{\rho}\right\}, C:=\bigcup_{\rho \in \tilde{T}}\left\{(C)^{\rho}\right\} .
\end{aligned}
$$

$\mathcal{T}:=E-P A^{\omega}+C$. The theory $\mathcal{T}$ is a formalization of simple type theory in the language of functionals of finite type without choice (see also Luckhardt (1975)).

$$
(A C !)^{1,0}-\text { qf. } \vdash \forall x^{1} \exists ! y^{0} A_{0}(x, y) \rightarrow \exists Y^{01} \forall x A_{0}(x, Y x) .
$$




\subsection{Lemma}

1) $W E-H A^{\omega}+A C \vdash b-A C$,

2) $W E-P A^{\omega}+b-A C \vdash C$.

Proof: 1) is trivial.

2) $W E-P A^{\omega} \vdash \forall x^{\rho} \exists n \leq 1\left(n={ }_{0} 0 \leftrightarrow A(x)\right) \Rightarrow$

$W E-P A^{\omega}+(b-A C)^{\rho, 0} \vdash \exists Y \leq_{0 \rho} \lambda v^{\rho} .1 \forall x^{\rho}(Y x=0 \leftrightarrow A(x))$.

\subsection{Application}

1) $\mathcal{M} \models \mathcal{T}+b-A C+D C$,

2) $\mathcal{M} \mapsto(A C !)^{1,0}-$ qf.,

3) $\mathcal{T}+b-A C+D C \nvdash(A C !)^{1,0}-\mathrm{qf}$.

Proof: 1) By $2.18 \mathcal{M}$ is a model of $E-P A^{\omega}$.

We show $\mathcal{M}=b-A C$ : Assume $Z \in \mathcal{M}$ and

$\forall x \in M_{\rho} \exists y \in M_{\tau}\left(y \leq_{\tau} Z x \wedge[A]_{\mathcal{M}}(x, y)\right)$. Using choice on the meta-level it follows that

$$
\exists Y \in M_{\tau}^{M_{\rho}} \forall x \in M_{\rho}\left(Y x \leq_{\tau} Z x \wedge[A]_{\mathcal{M}}(x, Y x)\right) .
$$

$Z \in M_{\tau \rho} \Rightarrow \exists Z^{*} \in M_{\tau \rho}: Z^{*} \operatorname{maj} Z \stackrel{Y \leq Z}{\Longrightarrow} Z^{*} \operatorname{maj} Y \in M_{\tau \rho}$.

$\mathcal{M} \models D C$ holds since $M_{\rho}^{M_{0}}=M_{\rho 0}$ (By $D C$ on the meta-level).

2) Assume $\mathcal{M} \models(A C)^{1,0}$-qf. By $(C)^{00}$ let $v \in \mathcal{M}$ be such that

(i) $\exists z^{0}\left(x^{00} z=0\right) \Leftrightarrow v x=0$ for all $x^{00}$. (AC $)^{1,0}$-qf. applied to $\forall x^{00} \exists z^{0}(v x=0 \Rightarrow$ $x z=0)$ yields

(ii) $\exists y \in M_{0(00)} \forall x^{00}[v x=0 \Rightarrow x(y x)=0]$. Since $y \in M_{0(00)}$ there exists a $y^{*} \in M_{0(00)}$ such that $y^{*} \operatorname{maj}_{0(00)} y$. From (i) and (ii) it follows that

$\forall m \in \omega(y(\overline{1, m}) \geq m)$. On the other hand one has $1^{00} \operatorname{maj}_{00} \overline{1, m}$ and therefore $y^{*} 1^{00} \geq y(\overline{1, m}) \geq m$ for all $m$, which is a contradiction. $(A C !)^{1,0}-\mathrm{qf}$. implies $(A C)^{1,0}$-qf. (relative to $\left.W E-P A^{\omega}\right)$ : Replace $A_{0}(x, y)$ by $A_{0}^{\prime}(x, y): \equiv A_{0}(x, y) \wedge$ $\forall \tilde{y}<y\left(\neg A_{0}(x, \tilde{y})\right)$

$\left(A_{0}^{\prime}\right.$ can also be expressed in a quantifier-free form in $\left.W E-P A^{\omega}\right)$. Therefore 2) follows.

3) follows immediatly from 1) and 2). 


\subsection{Remark}

In the context of set theory in a language with set variables instead of functional variables where functionals are identified with their graphs, $(A C !)^{1,0}$ is provable from the schema of comprehension formulated as the existence of the comprehending set (instead of its characteristic functional). In Feferman/Levy (1963) it is shown that dependent choice is not provable within $Z F$. This result uses P.Cohen's method of forcing.

As a final application, we show how $\mathcal{M}$ seperates different $\mu$-operators:

\subsection{Definition}

$$
\begin{gathered}
{ }^{\rho} \mu_{s} x^{0(\rho 0)} y^{\rho 0}:=\left\{\begin{array}{l}
\min n[x(\overline{y, n})<n] \text { if } \exists n(x(\overline{y, n})<n) \\
\text { undefined otherwise },
\end{array}\right. \\
{ }^{\rho} \mu_{1} x^{0(\rho 0)} y^{\rho 0}:=\left\{\begin{array}{l}
\min n\left[x(\overline{y, n})={ }_{0} x y\right] \text { if } \exists n\left(x(\overline{y, n})={ }_{0} x y\right), \\
\text { undefined otherwise },
\end{array}\right. \\
{ }^{\rho} \mu_{2} x^{0(\rho 0)} y^{\rho 0}:=\left\{\begin{array}{l}
\min n\left[x(\overline{y, n})={ }_{0} x(\bar{y} n * 1)\right] \text { if } \exists n\left(x(\overline{y, n})={ }_{0} x(\bar{y} n * 1)\right), \\
\text { undefined otherwise . }
\end{array}\right.
\end{gathered}
$$

\subsection{Remark}

The subscript $\mathrm{s}$ in the definition of ${ }^{\rho} \mu_{s}$ refers to Spector since $x(\overline{y, n})<n$ is the bar condition in his schema of bar recursion; see Spector (1962).

\subsection{Application}

${ }^{\rho} \mu_{s} \in \mathcal{M}$, but ${ }^{\rho} \mu_{1},{ }^{\rho} \mu_{2} \notin \mathcal{M}$ for each $\rho \in \tilde{T}$.

Proof: ${ }^{\rho} \mu_{s} x y$ is defined for all $x \in M_{0(\rho 0)}, y \in M_{\rho 0}$ :

By $\mathcal{M}$-definition and 2.17 there exists $x^{*} \in M_{0(\rho 0)}, y^{*} \in M_{\rho 0}$ such that $x^{*}$ maj $x$ and $y^{*}$ majy. Since $\forall n \in \omega\left(y^{*} \operatorname{maj}_{\rho 0} \overline{y, n}\right)$ it follows that $x^{*} y^{*} \geq x(\overline{y, n})$ for all $n \in \omega$ and hence $x\left(\overline{y, n_{0}}\right)<n_{0}$ for $n_{0}:=x^{*} y^{*}+1$ (see also Bezem (1985)). 
We now show that ${ }^{\rho} \mu_{s} \operatorname{maj} \mu_{s}$ : Assume again that $x^{*} \in M_{0(\rho 0)}, y^{*} \in M_{\rho 0}$ such that $x^{*} \operatorname{maj} x, y^{*} \operatorname{maj} y$. Then $\overline{y^{*}, n} \operatorname{maj}_{\rho 0} \overline{y, n}$ since $\operatorname{maj}_{\rho 0}$ is pointwise defined (!) and therefore $x^{*}\left(\overline{y^{*}, n}\right) \geq x(\overline{y, n})$ for all $\mathrm{n}$.

One concludes that ${ }^{\rho} \mu_{s} x^{*} y^{*} \geq{ }^{\rho} \mu_{s} x y$ and therefore ${ }^{\rho} \mu_{s}$ maj ${ }^{\rho} \mu_{s} \in \mathcal{M}$.

On the other hand, $\lambda x^{\rho} \cdot \mu_{i} x 1^{\rho 0} \notin M_{0}^{M_{\rho}} \supset M_{0 \rho}$ for $\mathrm{i}=1,2$ :

Assume $\rho=0 \rho_{k} \ldots \rho_{1}$ and $x \in M_{0}^{M_{\rho}}$ be defined by

$$
x y:=\left\{\begin{array}{l}
0 \text { if } \exists n \in \omega\left(y n O^{\rho_{1}} \ldots O^{\rho_{k}}={ }_{0} 0\right), \\
1 \text { otherwise }
\end{array}\right.
$$

for $y \in M_{\rho 0}$. Clearly $x \in M_{0(\rho 0)}$ since $1^{0(\rho 0)} \operatorname{maj} x$, and $x 1^{\rho 0}={ }_{0} x(\overline{1} n * 1)==_{0} 1$ but $x\left(\overline{1^{\rho 0}, n}\right)={ }_{0} 0$ for all $n \in \omega$. Therefore ${ }^{\rho} \mu_{1} x 1^{\rho 0}$ and ${ }^{\rho} \mu_{2} x 1^{\rho 0}$ are undefined, but $\mathcal{M}$ contains only total functionals.

\subsection{Corollary}

${ }^{\rho} \mu_{1}$ and ${ }^{\rho} \mu_{2}$ are not definable within $W E-H A^{\omega}+\mu_{s}$ where $W E-H A^{\omega}+\mu_{s}$ denotes the enlargement of $W E-H A^{\omega}$ by new constants ${ }^{\tau} \mu_{s}$ together with the defining axioms

$x\left(\overline{y, \mu_{s} x y}\right)<\mu_{s} x y$ and $x(\overline{y, n})<n \rightarrow \mu_{s} x y \leq n \quad\left(x^{0(\tau 0)}, y^{\tau 0}\right)$ for all types $\tau$.

\subsection{Remark}

In Kohlenbach (1990) it is proved that ${ }^{\rho} \mu_{s},{ }^{\rho} \mu_{i}$ are definable in $W E-H A^{\omega}+{ }^{\tau} \mu_{j}$ where $i, j=1,2$ and $\rho, \tau \in \tilde{T}$ arbitrary.

\subsection{Proposition}

There is no set-theoretic functional $\Phi^{0(0(00))}$ which majorizes $\lambda x^{0(00)} \cdot{ }^{0} \mu_{1} x 1^{00}(=$ $\lambda x^{0(00)} \cdot{ }^{0} \mu_{2} x 1^{00}$ ) on all primitive recursive arguments $x^{0(00)}$ (on which ${ }^{0} \mu_{1}$ and ${ }^{0} \mu_{2}$ are always defined!). In particular ${ }^{0} \mu_{1, t} \notin \mathcal{M}$ where

$$
{ }^{0} \mu_{1, t} x^{0(00)} y^{00}:=\left\{\begin{array}{l}
\min n\left[x(\overline{y, n})={ }_{0} x y\right] \text { if } \exists n\left(x(\overline{y, n})={ }_{0} x y\right) \\
0^{0} \text { otherwise. }
\end{array}\right.
$$


Proof: Assume that $\Phi m a j_{0(0(00))} \lambda x .{ }^{0} \mu_{1} x 1^{00}$ on prim. rec. arguments.

Define

$$
z_{n}^{0(00)} x^{00}:=\left\{\begin{array}{l}
0 \text { if } x n=0 \\
1 \text { otherwise }
\end{array}\right.
$$

$z_{n}$ is primitive recursive in $n$ (in the sense of $\widehat{P R}$ ) and $1^{0(00)} \operatorname{maj} z_{n}$ for all $n \in \omega$. Therefore $\Phi 1^{0(00)} \geq{ }^{0} \mu_{1} z_{n} 1^{00}$ for all $n \in \omega$. On the other hand, one has $\lim _{n \rightarrow \infty}{ }^{0} \mu_{1} z_{n} 1=\infty$ since $\mu_{1} z_{n} 1=n+1$ which is a contradiction.

\section{References}

\section{Bezem, M.A.}

Strongly majorizable functionals of finite type: a model for bar recursion containing discontinuous functionals. J. Symb. Logic 50,652-660 (1985).

\section{Bezem, M.A.}

Bar recursion and functionals of finite type. Dissertation, Utrecht (1986).

\section{Feferman, S.}

Theories of finite type. In: Barwise, J.(ed.) Handbook of mathematical logic (pp. 913-972). Amsterdam, North-Holland 1977.

\section{Feferman, S.,Levy, A.}

Independence results in set theory by Cohen's method II (abstract). Notices AMS 10,593 (1963).

\section{Friedrich, W.}

Spielquantorinterpretation unstetiger Funktionale in der höheren Analysis. Arch. Math. Logic $24,73-99$ (1984).

\section{Friedrich, W.}

Gödelsche Funktionalinterpretation unstetiger Funktionale der höheren Analysis. Zeitschr. f. math. Logik und Grundl. d. Math. 31 ,3-29 (1985).

\section{Gödel, K.}

Über eine bisher noch nicht benutzte Erweiterung des finiten Standpunktes. Dialectica 12,280-287 (1958).

\section{Howard, W.A.}

Hereditarily majorizable functionals of finite type. In: Troelstra A.S.(ed.)(1973). 


\section{Kleene S.C.}

Recursive functionals and quantifiers of finite types I. Trans. AMS 91,1-52 (1959).

\section{Kohlenbach, U.}

Theorie der majorisierbaren und stetigen Funktionale und ihre Anwendung bei der Extraktion von Schranken aus inkonstruktiven Beweisen: Effektive Eindeutigkeitsmodule bei besten Approximationen aus ineffektiven Eindeutigkeitsbeweisen. Dissertation, Frankfurt, pp. xxii+278 (1990).

\section{Luckhardt, $\mathbf{H}$.}

Extensional Gödel functional interpretation. A consistency proof of classical analysis. Springer Lecture Notes in Mathematics Vol. 306, Berlin Heidelberg New-York, Springer (1973).

\section{Luckhardt, $\mathbf{H}$.}

The real elements in a consistency proof for simple type theory I. In: Diller, J., Müller, G.H. (eds.) Proof theory symposium Kiel 1974. Springer Lecture Notes in Mathematics Vol. 500, Berlin Heidelberg New-York (1975).

\section{Schwichtenberg, $\mathbf{H}$.}

Einige Anwendungen von unendlichen Termen und Wertfunktionalen. Habilitationsschrift, Münster (1973).

\section{Spector, C.}

Provable recursive functionals of analysis: a consistency proof of analysis by an extension of principles formulated in current intuitionistic mathematics. In: J.C.E. Dekker (ed.) Recursive function theory. Proceedings of Symposia in Pure Mathematics, vol. 5, AMS, Providence, R.I.,pp. 1-27 (1962).

\section{Troelstra, A.S.}

(Ed.) Metamathematical investigation of intuitionistic arithmetic and analysis. Springer Lecture Notes in Mathematics, vol. 344, Berlin Heidelberg New-York (1973).

\section{Troelstra, A.S.}

Note on the fan theorem. J. Symb. Logic $39,584-596$ (1974).

\section{Troelstra, A.S.}

Some models for intuitionistic finite type arithmetic with fan functional. J. Symb. Logic 42,194-202 (1977). 